CANCER

\section{Early tumour growth halted}

Cells that eventually give rise to tumours attract immune cells that help to shield them from the body's defences. This may be one of the earliest events in tumour formation.

Bin Zhao at Zhejiang University in Hangzhou, China, and his colleagues studied liver cancer in mice and found that tumourinitiating cells (TICs) turn on production of Yes-associated protein (YAP). This molecule promotes cell proliferation and attracts immune cells called macrophages, which boost tumour growth. The TICs began doing this at an early stage of tumour growth, when they existed as single cells.

Blocking macrophage recruitment prevented TICs from developing into tumours and caused the immune system to eliminate them rapidly. The team also discovered YAP activation and macrophage recruitment in a small sample of precancerous lesions from human livers, suggesting that similar mechanisms might be involved in some human cancers.

Targeting YAP or macrophages could be a therapeutic strategy for liver cancer, the authors suggest. Genes Dev. http://doi.org/b2jc (2017)

\section{PLANETARY SCIENCE}

\section{Volcano gas makes worlds habitable}

Gases from erupting volcanoes could make planets beyond the Solar System warmer and hospitable to life, even if the planet is relatively distant from its host star.

Ramses Ramirez and Lisa Kaltenegger of Cornell

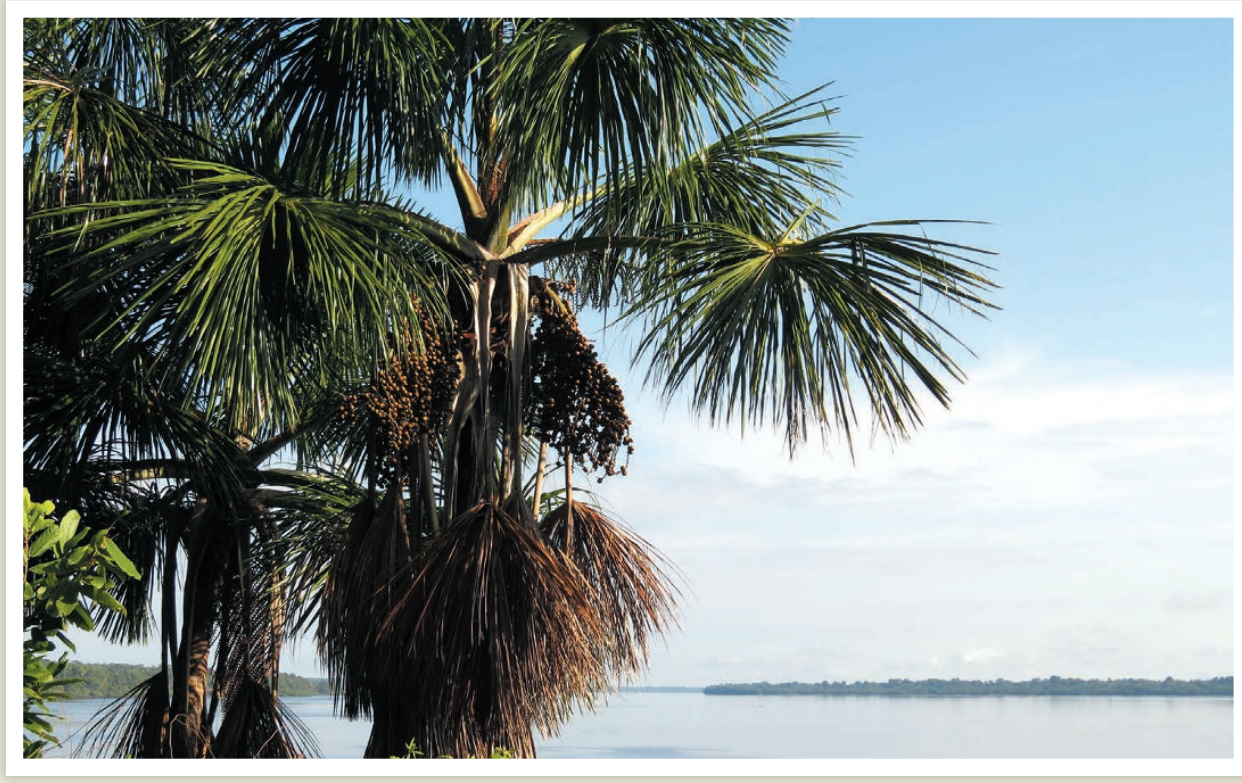

\title{
Early Amazonians left their mark
}

Woody plants such as the cocoa plant and Brazil nut tree that were domesticated by pre-Columbian peoples dominate the Amazon, suggesting that these populations had a strong influence on the ecosystem.

Carolina Levis at Wageningen University and Hans ter Steege at the Naturalis Biodiversity Center in Leiden, both in the Netherlands, and their colleagues looked at tree data from 1,170 Amazonian forest plots, and compared species prevalence with the locations of known archaeological sites dating to more than 500 years ago. They found that domesticated species such as the palm Mauritia flexuosa (pictured) were more common than other plants in and around such sites. The domesticated plants were also five times more likely to be highly dominant across the Amazon.

Measuring the prevalence of domesticated plants may help scientists to locate new archaeological sites, the authors suggest. Science 355, 925-931 (2017)
University in Ithaca, New York, used a climate model to study whether hydrogen spewing from volcanoes on exoplanets could build up in their atmospheres and trap heat. Such greenhouse warming could prevent water from freezing, potentially creating favourable conditions for life.

The authors found that if an exoplanet's atmosphere contained $50 \%$ hydrogen, the planet could remain habitable 1.3 to 1.6 times farther away from its star than if it was not warmed by volcanic gas. Astrophys. J. 837, L4 (2017)

\section{GENOMICS \\ Gene time bomb hurt mammoths}

Before becoming extinct, the last mammoths accumulated harmful genetic mutations that may have altered their behaviour and appearance.

Woolly mammoths (Mammuthus primigenius) disappeared from North America and Siberia 10,000 years ago, but small groups survived on islands until about 4,000 years ago. Rebekah Rogers and
Montgomery Slatkin at the University of California, Berkeley, compared two published genome sequences - one from a Siberian mainland specimen dated to 45,000 years ago, when mammoths numbered more than 12,000 , the other a 4,300-year-old specimen from Wrangel Island in the Arctic Ocean, which had a population of just 300 individuals.

They found that the island mammoth had more harmful mutations, including many gene deletions. The mutations may have affected 